University of South Carolina

Scholar Commons

3-1-2001

\title{
Quasirandom Distributed Gaussian Bases for Bound Problems
}

Sophya Garashchuk

University of South Carolina--Columbia, sgarashc@chem.sc.edu

John C. Light

Follow this and additional works at: https://scholarcommons.sc.edu/chem_facpub

Part of the Library and Information Science Commons

\section{Publication Info}

Published in Journal of Chemical Physics, Volume 114, Issue 9, 2001, pages 3929-3939.

http://jcp.aip.org/

(C) 2001 by American Institute of Physics

This Article is brought to you by the Chemistry and Biochemistry, Department of at Scholar Commons. It has been accepted for inclusion in Faculty Publications by an authorized administrator of Scholar Commons. For more information, please contact digres@mailbox.sc.edu. 


\title{
Quasirandom distributed Gaussian bases for bound problems
}

\author{
Sophya Garashchuk and John C. Light \\ James Franck Institute, University of Chicago, Chicago, Illinois 60637
}

(Received 13 June 2000; accepted 18 December 2000)

\begin{abstract}
We introduce quasirandom distributed Gaussian bases (QDGB) that are well suited for bound problems. The positions of the basis functions are chosen quasirandomly while their widths and density are functions of the potential. The basis function overlap and kinetic energy matrix elements are analytical. The potential energy matrix elements are accurately evaluated using few-point quadratures, since the Gaussian basis functions are localized. The resulting QDGB can be easily constructed and is shown to be accurate and efficient for eigenvalue calculation for several multidimensional model vibrational problems. As more demanding examples, we used a 2D QDGB-DVR basis to calculate the lowest 400 or so energy levels of the water molecule for zero total angular momentum to sub-wave-number precision. Finally, the lower levels of $\mathrm{Ar}_{3}$ and $\mathrm{Ne}_{3}$ were calculated using a symmetrized QDGB. The QDGB was shown to be accurate with a small basis. (C) 2001 American Institute of Physics. [DOI: 10.1063/1.1348022]
\end{abstract}

\section{INTRODUCTION}

The evaluation of highly excited vibrational states of polyatomic molecules and clusters is of interest for a number of fields. The theoretical spectra, when compared with experiment, yield information about the accuracy of the potential energy surfaces, predictions of levels not yet observed, information on the chaotic or regular character of the dynamics, etc. Most theoretical calculations on large systems now use direct product discrete variable representations (DVR), introduced in Refs. 1 and 2, and reviewed recently in Ref. 3, or DVR's in combination with basis representations for some coordinates. The most sophisticated calculations to date, such as those of Viel and Leforestier ${ }^{4}$ for HFCO, utilize sixdimensional DVR's of over $10^{7}$ points, truncated by potential energy to some 500000 points. The sophisticated adiabatic pseudo-spectral method due to Friesner ${ }^{5}$ was then used to obtain about 150 accurate eigenvalues and eigenstates. Other large systems such as the dimer of rigid water molecules have been solved by combinations of DVR's and coupled angular bases. ${ }^{6}$

Although DVR's greatly simplify the construction of Hamiltonian matrix elements and lead to a structured Hamiltonian which can be exploited in the solution, the sheer size of the basis, which scales as $n^{d}$, where $d$ is the dimension and $n$ is some average number of basis functions per dimension (say 10 or so) will pose a major challenge for systems with five or more atoms $(d \geqslant 9)$. We have thus begun looking for methods to define more compact multidimensional correlated bases for which the scaling might be more closely proportional to the number of desired eigenstates. Obviously if we could magically choose the eigenstates as the basis the problem would be solved.

Some time ago distributed Gaussian basis sets ${ }^{7,8}$ were introduced for multidimensional vibrational problems with semiclassical criteria governing the parameters of the Gaussians. Davis and Heller ${ }^{7}$ examined complex Gaussian basis sets distributed in phase space (Wigner distribution). Later we demonstrated that real distributed Gaussian basis sets (DGB's) (Ref. 8) gave comparable or improved efficiency and accuracy. In one dimension we made the distance between neighboring Gaussians proportional to the local de Broglie wavelength, and chose the exponents to give a desired average overlap. These were later used very effectively in conjunction with DVR's (usually in angles) for a number of triatomic systems. ${ }^{9-13}$ DGB were also used for distances $^{14,15}$ and for distances and angle. ${ }^{16}$

There were, however, some inconveniences associated with using even real DGB's which are not orthogonal. The orthogonalization of the basis, the problem of maintaining linear independence, and the choice of Gaussian centers and widths make them somewhat awkward to use, despite their high efficiency. However, optimization of DGB's for higher dimensional systems has not been investigated. As a first step in such an approach, we recently showed that fully optimized DGB's can be extraordinarily efficient and accurate for $1 \mathrm{D}$ systems. ${ }^{17}$ One interesting result was the demonstration that the most accurate results were obtained with very large condition numbers for the overlap matrix, S. A large condition number, the ratio of the largest eigenvalue to lowest eigenvalue, implies near linear dependence of the basis.

In this paper we look toward developing efficient DGB's for multidimensional systems. We look at three questions: the optimum or nearly optimum placement of DGB's in model potentials in one to three dimensions; the optimum condition numbers of the overlap matrix, and the scaling of the number of basis functions with the number of desired accurate solutions and with dimension. We build on the semiclassical criteria for placement of Gaussians proposed long ago, ${ }^{8}$ but look at quasirandom methods of placement which will be applicable to arbitrary dimensionality and potentials. We will first apply the quasirandom distributed Gaussian basis (QDGB) approach to problems with simple potentials, but we then do an accurate DVR-QDGB calculation of the vibrational levels of $\mathrm{H}_{2} \mathrm{O}(J=0)$ up to about $28000 \mathrm{~cm}^{-1}$. The DVR-QDGB appears to be substantially 
more accurate than the earlier DVR-DGB calculation ${ }^{11}$ for the same basis size. Finally, we use a QDGB for the rare gas trimers $\left(\mathrm{Ar}_{3}\right.$ and $\left.\mathrm{Ne}_{3}\right)$ using bond distance coordinates. Again the quasirandom placement is shown to be more efficient than the recent DGB on a grid calculation of Ref. 15.

In the next section we "optimize" the placement of the centers of the Gaussian basis functions and their widths in 1D potentials and study the effects of scaling the Gaussian widths (varying the condition number of $\mathbf{S}$ ) on the relative accuracy of vibrational eigenvalues. Both the "optimal" widths and the density of Gaussians are found to be the linear functions of the potential. We also found that a range of fairly large condition numbers of the overlap matrix give accurate energy eigenvalues. In Sec. III we transfer our experience with the 1D "optimized" Gaussian bases into many dimensions and construct DGB's using QDGBs whose widths and density are linear functions of the potentials. We analyze the effectiveness and the performance of the quasirandom DGB for 1D-3D Morse potentials and for the Henon-Heiles potential. The accuracy of the numerical quadrature integration for the potential matrix elements and the dependence on the two parameters determining the basis are also tested. In Sec. IV A we present the results for $\mathrm{H}_{2} \mathrm{O}$ $(J=0)$, using a combination of QDGB for the two radial coordinates and a DVR for the angular coordinate. In Sec. IV B we present the 3D QDGB results for $\mathrm{Ne}_{3}$ and $\mathrm{Ar}_{3}$. Section V concludes.

\section{OPTIMIZED GAUSSIAN BASIS SET IN ONE DIMENSION}

For 1D systems and small Gaussian basis sets a full optimization can be carried out, ${ }^{17}$ where it was shown that the lower eigenvalues are slowly varying with basis parameters near the optimum basis. However the full optimization method scales as the fifth power of the basis size. We seek, therefore, a more practical approach to finding a good if not optimum Gaussian bases.

Our initial goal is to construct an efficient basis consisting of real normalized Gaussians, $\left\{g_{i}\right\}$, in coordinate space,

$$
g_{i}=\left(2 \alpha_{i} / \pi\right)^{1 / 4} \exp \left(-\alpha_{i}\left(x-x_{i}\right)^{2}\right)
$$

for a one-dimensional Hamiltonian,

$$
\hat{H}=-\frac{1}{2} \frac{d^{2}}{d x^{2}}+V(x)
$$

We want the basis to describe adequately the energy eigenfunctions below a certain cutoff energy $E_{\text {cut }}$. The Gaussian basis is not orthogonal, and in order to find the eigenvalues of $\hat{H}$ one has to construct the overlap matrix $\mathbf{S}$ with the elements $S_{i j}=\left\langle g_{i} \mid g_{j}\right\rangle$, the Hamiltonian matrix $\mathbf{H}$ with the elements $H_{i j}=\left\langle g_{i}|\hat{H}| g_{j}\right\rangle$ and to solve the generalized eigenvalue problem

$$
\mathrm{HB}=\mathrm{SBE} .
$$

The overlap matrix elements

$$
S_{i j}=\sqrt{\frac{2 \sqrt{\alpha_{i} \alpha_{j}}}{\alpha_{i}+\alpha_{j}}} \exp \left(-\frac{\alpha_{i} \alpha_{j}}{\alpha_{i}+\alpha_{j}}\left(x_{i}-x_{j}\right)^{2}\right),
$$

and the kinetic energy matrix elements

$T_{i j}=\left\langle g_{i}\left|-\frac{1}{2} \frac{d^{2}}{d x^{2}}\right| g_{j}\right\rangle=\left(\frac{\alpha_{i} \alpha_{j}}{\alpha_{i}+\alpha_{j}}-2\left(\frac{\alpha_{i} \alpha_{j}}{\alpha_{i}+\alpha_{j}}\left(x_{i}-x_{j}\right)\right)^{2}\right) S_{i j}$

have simple analytic forms. The potential matrix elements $V_{i j}=\left\langle g_{i}|V| g_{j}\right\rangle$ can be accurately calculated using low order Gauss-Hermite quadrature, since the basis functions are localized and a product of two Gaussian basis functions is a single Gaussian. The diagonal matrix $\mathbf{E}$ gives the eigenvalues of the Hamiltonian $\hat{H}$ and the columns of the matrix $\mathbf{B}$ are the respective eigenvectors.

The $i$ th diagonal matrix element of the kinetic energy is $T_{i i}=\alpha_{i} / 2$, so we expect the optimal $\alpha_{i}$ to be proportional to the maximal kinetic energy at the center $x_{i}$ of a corresponding Gaussian,

$$
\alpha_{i} \sim\left(E_{\text {cut }}-V\left(x_{i}\right)\right) .
$$

Semiclassical spacing of the Gaussian basis ${ }^{8}$ suggests their separation should be proportional to the de Broglie wavelength, and thus the density to be proportional to

$$
\rho \sim\left(E_{\text {cut }}-V\left(x_{i}\right)\right)^{\gamma},
$$

with $\gamma=1 / 2$. We show below that a linear dependence $(\gamma=1)$ is perhaps preferable.

These criteria yield basis functions which are narrower in regions of low potential and, consequently, the centers may be more dense where the de Broglie wavelength is small, and they will be broader and further apart in the regions of high potential.

To verify that this is a good choice, we show that it results from the minimization of the following functional,

$$
\widetilde{F}=\operatorname{Tr}(\mathbf{H})-\lambda \sum_{i j, i \neq j} \frac{S_{i j}}{1-S_{i j}}
$$

with respect to the positions of Gaussians $\left\{x_{i}\right\}$ and to their widths $\left\{\alpha_{i}\right\}$.

No functional form of $\alpha_{i}$ or the density is specified at this stage, since the widths and positions are to be found variationally. The functional includes the full trace of the Hamiltonian and the artificial repulsion term whose strength is determined by the parameter $\lambda$.

While the functional is ad hoc, it is based on physical considerations and does yield an excellent basis. If the basis is orthogonal, the second term is zero and the trace of the Hamiltonian will be minimized. For a normed but nonorthogonal basis, the minimum of the $\operatorname{tr}[\mathrm{H}]$ term alone would yield $\mathrm{N}$ replicas of the ground state, $\operatorname{tr}[\mathrm{H}]=\mathrm{N} \epsilon_{o}$. The second term of the functional forces linear independence of the functions and leads to acceptable bases. The repulsion term prevents Gaussians from being placed too close to each other during the minimization procedure and guarantees that the overlap matrix $\mathbf{S}$ is not singular. The parameter $\lambda$ is nonvariational, and minimization of this functional will not produce the optimal Gaussian basis. However, the minimization 
procedure generates a basis that becomes very efficient after Gaussian widths are scaled uniformly to optimize the accuracy with the full Hamiltonian diagonalization.

We apply the outlined procedure to the calculation of all the bound energy levels of a one-dimensional Morse oscillator,

$$
V(x)=D(\exp (-w x)-1)^{2} .
$$

The dissociative character of the potential requires the introduction of a finite coordinate range where Gaussians are be placed. To limit the range to $x_{i}<x_{\max }$ we simply add a step potential $V_{\text {step }}=V_{0} \theta\left(x_{\max }-x\right)$. We use the Morse potential B in Ref. 8 with parameters $D=12.0$ and $w=0.2041241$ which supports 24 bound states. The amplitude of the step potential is taken $V_{0}=25.0$, well above the energy cut-off. The highest true bound state wave function has its broad maximum at $x \approx 35.0$; thus we set $x_{\max }=70$.

We found that the range of the nonvariational parameter $\lambda$ for which the minimization procedure converges is $[0.5,1.5]$. The optimization of $\left\{\alpha_{i}\right\}$ and $\left\{x_{i}\right\}$ (with $\lambda=1.0$ ) produces the "expected" distribution of Gaussian centers and widths, with the reciprocal condition number $(\mathrm{RCN})$ of the overlap matrix $\mathbf{S}$, which is the ratio of the smallest eigenvalue to the largest eigenvalue, on the order of $10^{-5}$. This $\mathrm{RCN}$ is orders of magnitude higher than the minimum required to yield stable numerical inversion. Scaling of all $\alpha_{i}$ by a constant, such that the RCN of the overlap matrix is reduced to $10^{-9}-10^{-12}$, increases the accuracy of the energy levels by about three orders of magnitude. We find that if the RCN is still smaller the eigenvalues of $\mathbf{H}$ increase.

For the very small RCN's the generalized eigenvalue problem may be solved by singular value decomposition which removes eigenvectors of $\mathbf{S}$ with very small eigenvalues. When this was applied to the overlap matrices with RCNs in the range $\mu=10^{-15}-10^{-13}$, we found that removing these few orthogonalized basis functions also increases the eigenvalues of $\mathbf{H}$. Thus, there is an optimal range of 2-4 orders of magnitude for the RCN, that yields the lowest eigenvalues of $\mathbf{H}$. We found this to be true for higher dimensions and all potentials examined. The RCN is adjusted by scaling of the widths $\alpha_{i}$ for all basis functions $g_{i}$ by the same factor.

This procedure, which produces an extremely efficient basis, permits us to examine the dependence of the widths and density on the potential. In Fig. 1 we plot the width parameters $\alpha_{i}$ and the density of Gaussian centers, defined as $\rho\left(x_{i}\right)=0.5\left(x_{i+1}-x_{i-1}\right)^{-1}$, as functions of the Gaussian center position. Both functions, the width and the density, can be accurately fit by linear functions of the potential energy, $f(x)=a_{0}\left(a_{1}-V(x)\right)$.

Figure 2 shows the relative accuracy of the energy levels on a logarithmic scale for $\left\{x_{i}\right\}$ and $\left\{\alpha_{i}\right\}$ obtained by the minimization of the functional and after scaling all $\alpha_{i}$ by 0.4 and by 0.13 yielding RCN's of the overlap matrix of $\mu$ $=0.00435, \mu=1.92 \times 10^{-6}$, and $\mu=1.47 \times 10^{-13}$. Relative accuracy is defined as

$$
\left(E_{i}-E_{i, \text { exact }}\right) / E_{i, \text { exact }} .
$$

Thus, we obtained all 24 energy levels using 48 Gaussian basis functions with relative accuracy better than $10^{-5}$, absolute accuracy better than $10^{-4}$. This gives a ratio of just 2 basis functions per accurate eigenvalue. The efficiency and accuracy of this basis with density scaling linearly with the potential $(\gamma=1)$ is nearly identical to that found earlier ${ }^{8}$ with semiclassical spacing $(\gamma=0.5)$. Note that the density is determined by minimizing the functional and was not imposed.

In Fig. 3 we show the analytical and numerical eigenfunctions for the highest vibrational level, which requires the Gaussians to be placed far into the dissociation region. A uniform grid of identical Gaussians with optimized exponents is much less efficient, requiring more than twice as many basis functions to obtain comparable accuracy.

To verify the dependence on potential of the "optimized" Gaussian parameters, we repeat the same variational procedure to obtain the basis set for the Gaussian double well potential as in ${ }^{8}$

$V(x)=-D\left(\exp \left(-w\left(x-x_{0}\right)^{2}\right)+\exp \left(-w\left(x+x_{0}\right)^{2}\right)\right)$

with the parameters $D=12.0, w=0.1$, and $x_{0}=5.0$. This potential also supports 24 bound states.

We used a basis of 54 Gaussians (without taking into account the symmetry of the potential). After uniform scaling of the exponents to yield the RCN of $10^{-9}$, we obtained all the vibrational levels with the relative accuracy better than $10^{-5}$. (Comparable accuracy requires 250 uniformly distributed Gaussians of equal width.) The distribution of $\alpha_{i}$ and the density of centers were once again accurately fit with linear functions of the potential energy, as shown in Fig. 4.

Full optimization of $\widetilde{F}$ for large multidimensional bases may be impractical. However, these results of the optimization of one dimensional bases using Eq. (8) suggest that after scaling, the choice given by Eq. (6) for the basis function width $\left\{\alpha_{i}\right\}$ and by Eq. (7) for the distribution of Gaussians

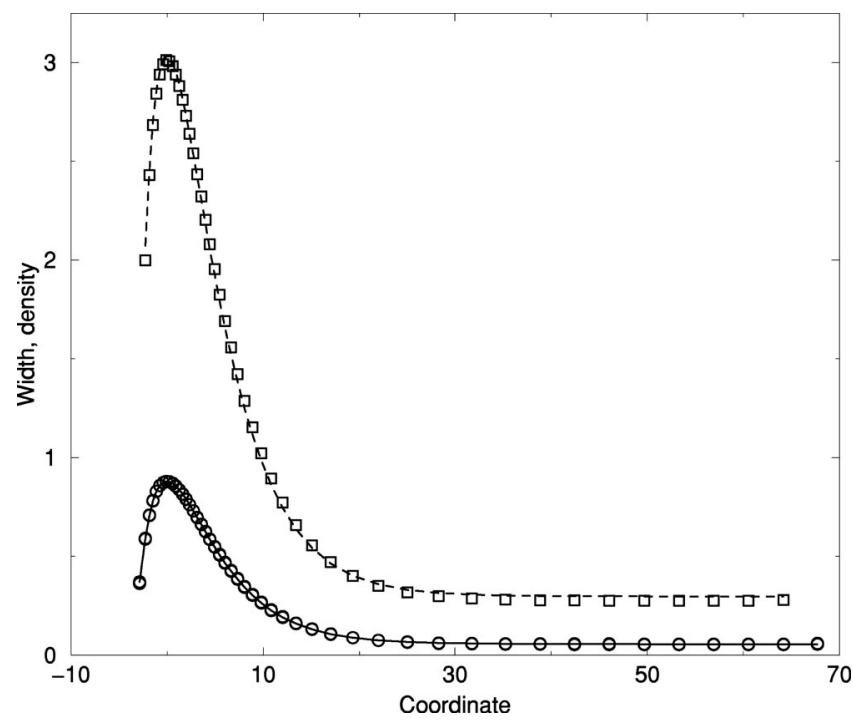

FIG. 1. The widths, $\left\{\alpha_{i}\right\}$, and the density of Gaussians as a function of their centers, $\left\{x_{i}\right\}$, for the Morse oscillator: circles mark $\alpha_{i}$ found from the minimization of the functional $\widetilde{F}$, Eq. (8); solid line is the fit of $\alpha_{i}$ with the linear function of the potential; squares mark the optimized density; and the dashed line is its linear fit. 


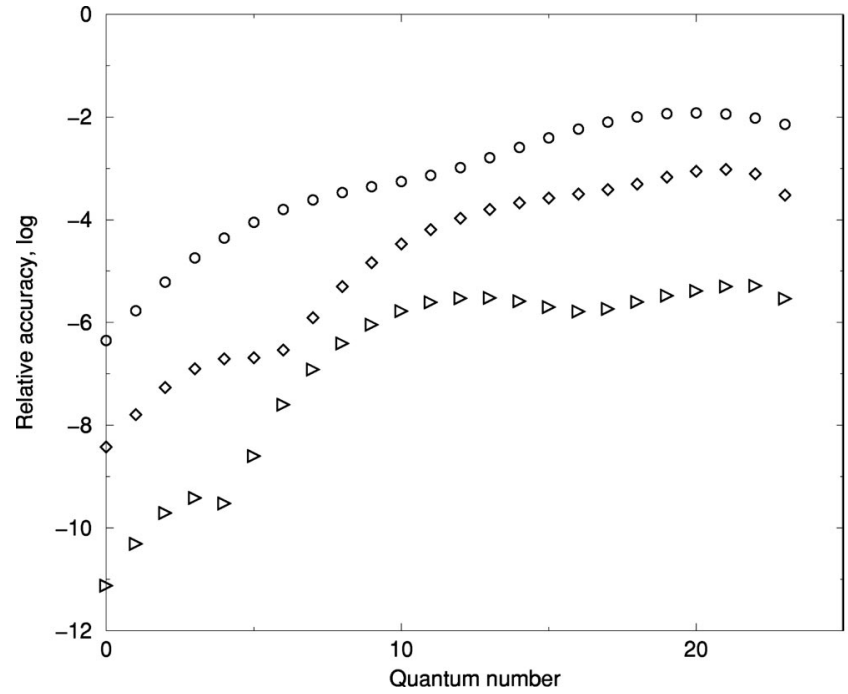

FIG. 2. Logarithm of the relative accuracy of the levels of the Morse oscillator, plotted as a function of the quantum number. The energy levels are calculated with the optimized and scaled basis function parameters for several reciprocal condition numbers $\mu$ of the overlap matrix $\mathbf{S}$ : circles, diamonds, and triangles correspond to $\mu=4.35 \times 10^{-3}, 1.92 \times 10^{-6}, 1.47$ $\times 10^{-13}$, respectively.

(with $\gamma=1$ ) will produce efficient basis sets in higher dimensions. Therefore for multidimensional problems we will use the functional form of Eqs. (6) and (7) for the width and density of Gaussians and use the RCN of the overlap matrix as a criterion for choosing the overall scaling factor of the widths $\alpha_{i}$.

\section{QUASIRANDOM DISTRIBUTION OF GAUSSIANS}

As was found previously, DGB's based on semiclassical considerations $^{9-12}$ are difficult to construct in more than one dimension, and, of course, the optimization procedure of Sec. II becomes expensive, if feasible at all. Thus, we take

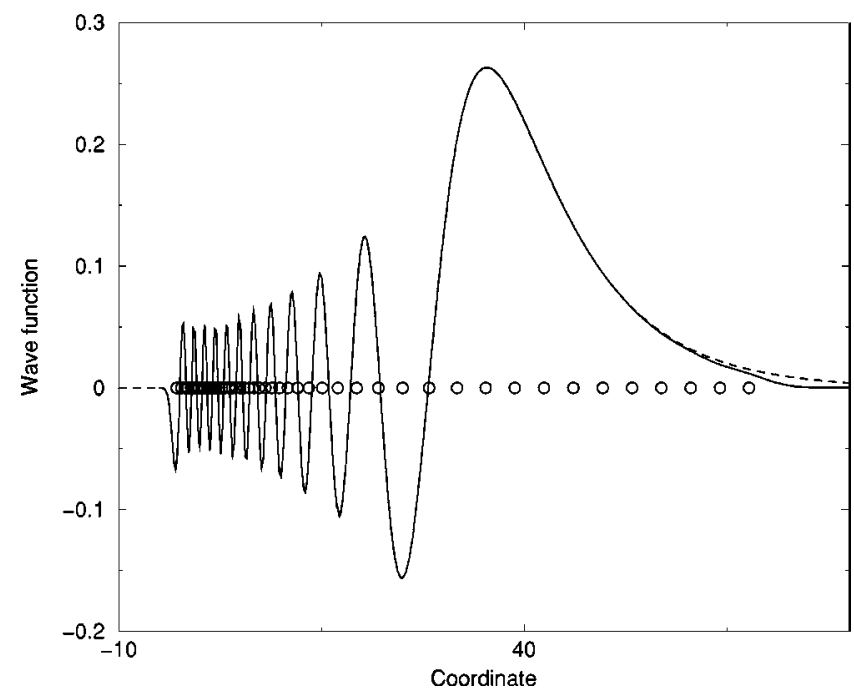

FIG. 3. The eigenfunction of the highest, $n=23$, energy level for the Morse potential in coordinate space: dashed line shows the analytical result and the solid line shows the numerical eigenfunction corresponding to the most accurate calculation in Fig. 2. The centers of basis functions (circles) are also shown.

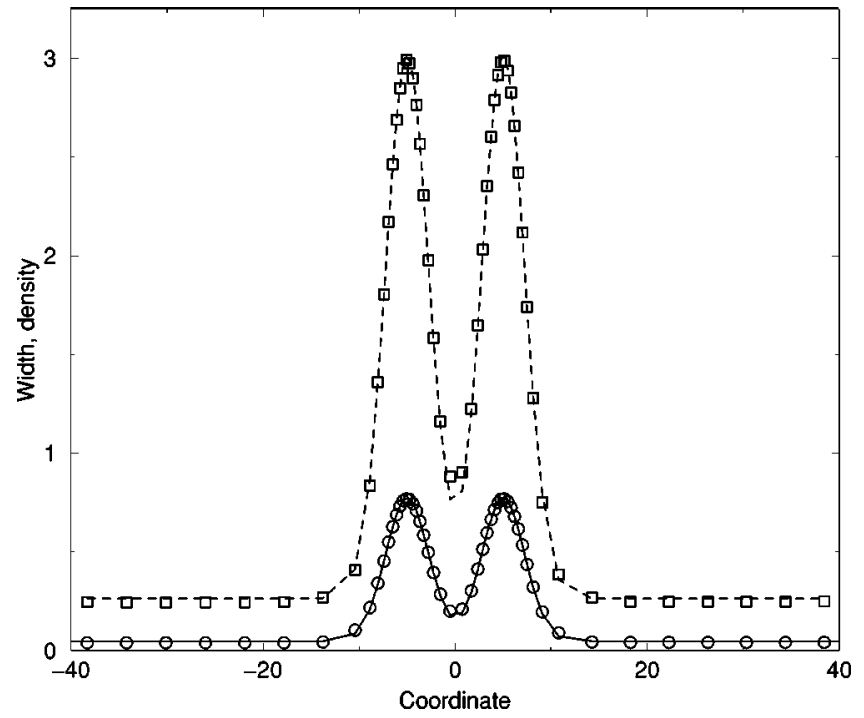

FIG. 4. The widths, $\left\{\alpha_{i}\right\}$, and the density of Gaussians as a function of their centers, $\left\{x_{i}\right\}$, for the double well potential: circles mark the optimized $\alpha_{i}$; solid line is the fit of $\alpha_{i}$ with the linear function of the potential; squares mark the optimized density; and the dashed line is its linear fit.

advantage of the simple functional form of the basis function parameters, a linear dependence on the potential, which yielded excellent results above after scaling of the widths.

For multidimensional problems we wish to use a DGB with the appropriate varying density of centers. Since this cannot be done simply on a grid, we will use a quasirandom procedure to distribute the Gaussian basis functions with potential-dependent density and widths within the energy contour $V\left(\mathbf{x}_{i}\right)<E_{\text {cut }}$. (Here $\mathbf{x}$ is the coordinate vector in the multidimensional space.) Thus we take

$g_{i}(\mathbf{x})=\exp \left(-\left(\mathbf{x}-\mathbf{x}_{i}\right)^{T} \mathbf{A}_{i}\left(\mathbf{x}-\mathbf{x}_{i}\right)\right)\left(2^{N} \operatorname{det}\left(\mathbf{A}_{\mathbf{i}}\right) / \pi^{N}\right)^{1 / 4}$.

The width parameters may be a matrix $\mathbf{A}_{i}$ with or without off-diagonal elements, in general. We consider $\mathbf{A}_{i}$ to be a diagonal matrix with equal elements $\alpha_{i}$ for Cartesian coordinates. We choose the scaled widths of Gaussians according to $\mathrm{Eq}$ (6) (for simplicity the minimum of the potential is taken to be zero),

$$
\alpha_{i}=c m_{i}\left(E_{\mathrm{cut}}-V\left(\mathbf{x}_{i}\right)+\Delta\right)
$$

with the same value of $c$ for all functions and dimensions chosen to yield the RCN of the overlap matrix on the order of $10^{-6}-10^{-14}$. This is consistent with the results of optimization for the one-dimensional problems of Sec. II.

The density of centers is also a linear function of the potential,

$$
\rho(\mathbf{x}) \sim E_{\text {cut }}-V\left(\mathbf{x}_{i}\right)+\Delta .
$$

The parameter $\Delta$ defines the ratio of the maximal $\alpha_{i}$ to its minimal value. The limit of large $\Delta, \Delta \gg E_{\text {cut }}$ corresponds to a uniformly dense quasirandom distribution of Gaussians of equal width. Small values of $\Delta, \Delta<E_{\text {cut }}$, correspond to a distribution where the density and the width of the basis functions are determined by the potential as in the "optimal' 'basis of Sec. II. A suitable value of $c$ to produce a good $\mathrm{RCN}$ is chosen after a few tries. The parameter $c$ can be 
estimated from the lowest eigenvalue as $c \sim E_{0} / E_{\text {cut }}$, or from a problem with a reduced $E_{\text {cut }}$ and a small number of Gaussians. The procedure has only two parameters and produces an efficient correlated basis for multidimensional systems.

In multidimensional systems we wish to distribute the Gaussian basis functions with varying density. This can be done with a random procedure accepting Gaussian centers with a probability proportional to the desired density and rejecting them if they exceed a maximum overlap with other members of the basis. A more efficient procedure is to use a quasirandom (or subrandom) sequence of numbers rather than random numbers. This has the advantage that the points $\mathbf{x}_{i}$ in the "random" sequence are generated as far apart as possible for a given density, thus reducing the probability that two points will be rejected because of too much overlap. (This greatly increases the efficiency of accepting a new basis function into the basis set.) The convergence of eigenvalues with respect to the number of basis functions can be monitored while the sequence is being generated.

We used the Sobol sequence to generate quasirandom points. ${ }^{18}$ The density of points can be modified according to Eq. (14) with the rejection method; ${ }^{18}$ the point $\mathbf{x}_{i}$ is accepted if

$$
\left[\frac{E_{\mathrm{cut}}-V\left(\mathbf{x}_{i}\right)+\Delta}{E_{\mathrm{cut}}+\Delta}\right]^{\gamma}>b_{i}
$$

where numbers $b_{i}=[0,1]$ belong to an independent sequence of random numbers. The choice of $\gamma=1$ is the linear relationship found above. $\left(\gamma=0.5\right.$ is the semiclassical choice ${ }^{8}$ tested below.) We will refer to the basis constructed in this way with $\gamma=1$ as the nonuniform quasirandom distributed Gaussian basis, QDGB.

To test the effect of the distribution of the basis function widths and density we compare with two other bases of Gaussians. One, with Gaussians of equal width distributed quasirandomly with the uniform density will be referred to as "uniform quasirandom DGB." The other is a uniform grid of Gaussians of equal width. Both are optimized with respect to the width parameter of the Gaussians.

\section{A. The one-dimensional Morse potential}

As a first test we look at the performance of quasirandom DGB for a one-dimensional Morse oscillator of Sec. II,

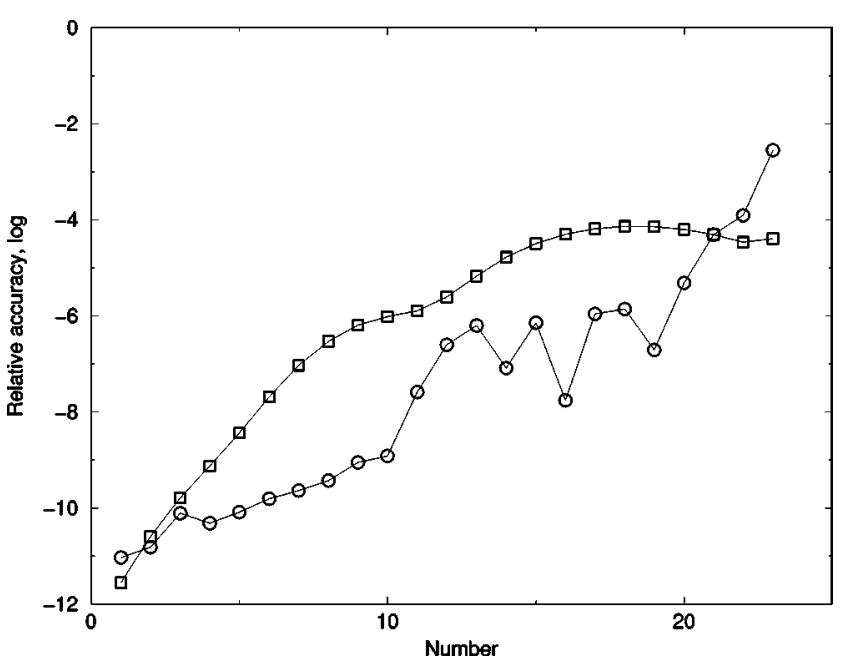

FIG. 5. Logarithm of the accuracy of the energy levels for one-dimensional Morse potential calculated with nonuniform QDGB of 48 basis functions [Eq. (15)] is shown with circles. The same, obtained with 80 Gaussians distributed uniformly on a grid, is shown with squares.

defined by Eq. (9). We look at the accuracy of the lowest 23 eigenvalues with the energies below 11.99 a.u. The energy levels are calculated with 48 Gaussians with widths that depend on the potential energy of their centers according to Eq. (13) and distributed quasirandomly using the rejection method (QDGB), Eqs. (14) and (15). We compare with 80 Gaussians of equal width distributed on a grid. The energy cutoff is the same for all basis sets, $E_{\text {cut }}=11.99$ a.u., and $\Delta$ $=0.1$ a.u. The exponent scaling parameter $c$ for the QDGB, as defined in Eq. (15) and the width of Gaussians for the grid basis, yielding the most accurate energy levels, were 0.164025 and 1.62 yielding the RCNs of the overlap matrices $1.6 \times 10^{-13}$ and $2.6 \times 10^{-5}$ for the QDGB and the basis set on a grid, respectively. The relative accuracy of the energy levels is shown in Fig. 5. The 48 Gaussians generated with the rejection method give accuracy which is better by at least one order of magnitude for 16 out of 23 levels, when compared to the calculation with 80 Gaussians on the grid.

The QDGB depends on two parameters $-\Delta$, controlling the sensitivity of the widths to the potential, and thus the variations of the density of the basis functions with potential, and $c$, which scales the widths $\alpha_{i}$ of all the Gaussians and controls the overlap of basis functions. The dependence of

TABLE I. The numerical parameters used to calculate the energy levels for system of Morse oscillators given by Eqs. (9), (16), and (17). The dissociation energy is $D=12.0$.

\begin{tabular}{|c|c|c|c|c|c|c|}
\hline \multirow{2}{*}{\multicolumn{2}{|c|}{$\begin{array}{l}\text { Frequency } \\
\text { basis set type }\end{array}$}} & \multicolumn{2}{|c|}{$w_{x}=0.2041241$} & \multicolumn{3}{|c|}{$w_{y}=0.18371169 \quad w_{z}=0.16329928$} \\
\hline & & $N^{\text {levels }}$ & $N^{\text {Gaus }}$ & $E_{\text {cut }}$ & Width, $c$ & $\mathrm{RCN}$ \\
\hline 1 & grid & 23 & 48 & 11.99 & 1.62 & $2.6 \times 10^{-5}$ \\
\hline 1 & nonuniform & 23 & 48 & 11.99 & 0.164025 & $1.6 \times 10^{-13}$ \\
\hline 2 & grid & 122 & 482 & 11.5 & 1.28 & $6.4 \times 10^{-8}$ \\
\hline 2 & uniform & 122 & 482 & 11.5 & 0.832 & $3.9 \times 10^{-10}$ \\
\hline 2 & nonuniform $\gamma=1$ & 122 & 482 & 11.5 & 0.12005 & $1.2 \times 10^{-12}$ \\
\hline 2 & nonuniform $\gamma=1 / 2$ & 122 & 482 & 11.5 & 0.12005 & $5.2 \times 10^{-11}$ \\
\hline 3 & grid & 124 & 939 & 7.4 & 1.0 & $7.7 \times 10^{-8}$ \\
\hline 3 & uniform & 124 & 939 & 7.4 & 0.704 & $1.1 \times 10^{-8}$ \\
\hline 3 & nonuniform & 124 & 939 & 7.4 & 0.6336 & $9.2 \times 10^{-12}$ \\
\hline
\end{tabular}




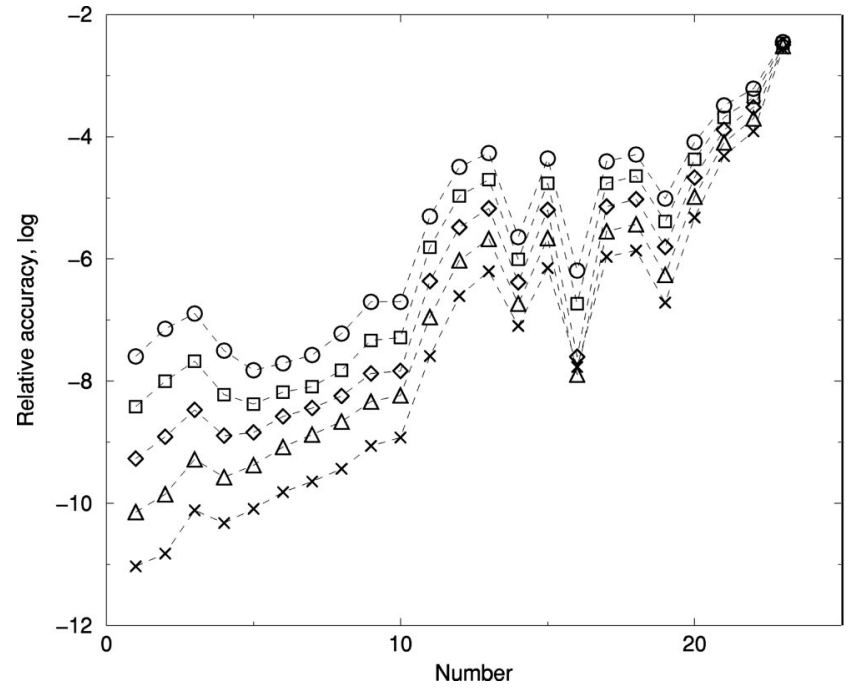

FIG. 6. The dependence of the accuracy of the energy levels for onedimensional Morse potential, calculated with uniform QDGB of 48 basis functions, on their width (parameter $c$ ): circles, squares, diamonds, triangles, and crosses correspond to values of $c$ being equal to $0.25,0.225$, $0.2025,0.18225$, and 0.164025 yielding the overlap matrix reciprocal condition numbers of $1.55 \times 10^{-10}, 3.06 \times 10^{-11}, 5.67 \times 10^{-12}, 9.87 \times 10^{-13}$, and $1.62 \times 10^{-13}$, respectively. The ordinate is the logarithm of the relative accuracy of the energy levels.

the energy levels on the overall scaling of Gaussian width through the parameter $c$ (or on the RCN of the overlap matrix) is shown in Fig. 6. The RCN changes by three orders of magnitude from $10^{-10}$ to $10^{-13}$, which affects the accuracy of the energy levels. However overall accuracy remains better than $10^{-4}$ for 20 out of 23 levels for all calculations and the accuracy of the remaining 3 levels depends on the RCN only weakly, being more strongly affected by the energy cutoff parameter $E_{\text {cut }}$. The dependence of the energy levels on the parameter $\Delta$ is shown in Fig. 7. Calculations per-

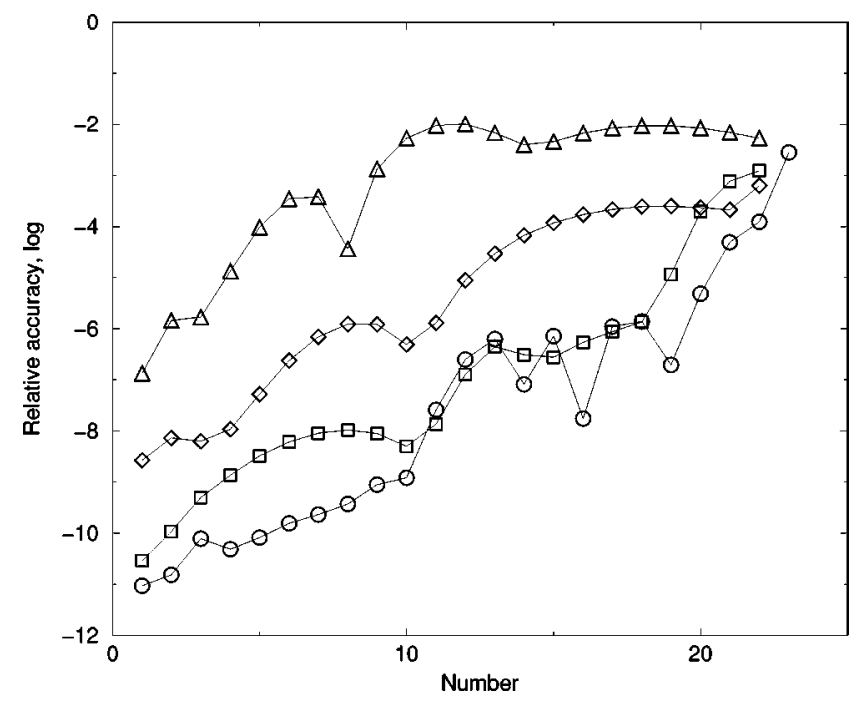

FIG. 7. The dependence of the accuracy of the energy levels for onedimensional Morse potential, calculated with uniform QDGB of 48 basis functions, on the parameter $\Delta$ : circles, squares, diamonds, and triangles correspond to $\Delta$ being equal to $0.1,1.0,6.0$, and 12.0 with the values of $c$ being equal to $0.164025,0.13122,0.054675$, and 0.0405 , respectively. The ordinate is the logarithm of the relative accuracy of the energy levels.
TABLE II. The difference in the energy levels for the 1D Morse potential calculated with the analytical potential matrix elements $V_{i j}$ and with the numerically evaluated $V_{i j}$ using one-, two-, and three-point Gauss-Hermite quadratures.

\begin{tabular}{cccc}
\hline \hline Level, $N$ & One-point & Two-point & Three-point \\
\hline 1 & 0.051864 & 0.000264 & 0.0000005 \\
2 & 0.051675 & 0.000273 & 0.0000006 \\
3 & 0.051380 & 0.000283 & 0.0000007 \\
4 & 0.050933 & 0.000293 & 0.0000007 \\
5 & 0.050288 & 0.000302 & 0.0000008 \\
6 & 0.049401 & 0.000310 & 0.0000009 \\
7 & 0.048232 & 0.000316 & 0.0000010 \\
8 & 0.046749 & 0.000320 & 0.0000010 \\
9 & 0.044929 & 0.000322 & 0.0000011 \\
10 & 0.042758 & 0.000321 & 0.0000012 \\
11 & 0.040228 & 0.000317 & 0.000001 \\
12 & 0.037338 & 0.000309 & 0.0000013 \\
13 & 0.034088 & 0.000297 & 0.0000013 \\
14 & 0.030487 & 0.000280 & 0.0000014 \\
15 & 0.026578 & 0.000257 & 0.0000014 \\
16 & 0.022348 & 0.000228 & 0.0000014 \\
17 & 0.017856 & 0.000191 & 0.0000014 \\
18 & 0.013210 & 0.000144 & 0.0000014 \\
19 & 0.008370 & 0.000082 & 0.0000012 \\
20 & 0.003594 & 0.000001 & 0.0000007 \\
21 & -0.000966 & -0.000119 & -0.0000007 \\
22 & -0.003225 & -0.000211 & -0.0000026 \\
23 & -0.003656 & -0.000324 & -0.0000154 \\
\hline \hline
\end{tabular}

formed with the smallest $\Delta$, that was about $1 \%$ of $E_{\text {cut }}$, i.e., the most sensitive to the potential, gives the most accurate results. The choice of $\Delta$ of about $10 \%$ and $50 \%$ of $E_{\text {cut }}$ also give quite accurate results, while $\Delta \approx E_{\text {cut }}$ gives significantly lower accuracy for all energy levels.

We also examine the accuracy of the numerical evaluation of the potential matrix elements for the Morse potential. Our basis functions are localized, but fairly strongly overlapping. We use Gauss-Hermite quadratures, since a product of two Gaussians is a Gaussian. In Table II we compare the energy levels if one-, two-, and three-point quadratures are used to compute the potential matrix elements. One can see that three-point quadrature gives accurate results, basically to six significant figures, and even a two-point quadrature might be sufficient if lower accuracy $\left(10^{-4}\right.$ in this example) is acceptable. We note that the accuracy of low order Gaussian quadratures depends on both the potential and the width of the Gaussians, and must be checked.

\section{B. Two and three dimensional systems}

The QDGB method can easily be extended to two and higher dimensional systems. We test the performance of three basis sets for two and three-dimensional Morse oscillators; QDGB's where positions of Gaussians are chosen quasirandomly with the density $\rho\left(\mathbf{x}_{i}\right) \sim\left(E_{\text {cut }}-V\left(\mathbf{x}_{i}\right)+\Delta\right)^{\gamma}$ with $\gamma=1.0,0.5$; a uniform width quasirandom basis and the uniform basis on a grid. All bases are truncated to include only Gaussians with centers within the energy contour $\mathrm{E}_{\text {cut }}$. The oscillators in the Hamiltonian are uncoupled for the sake of comparison with analytical energy levels, but nothing in 


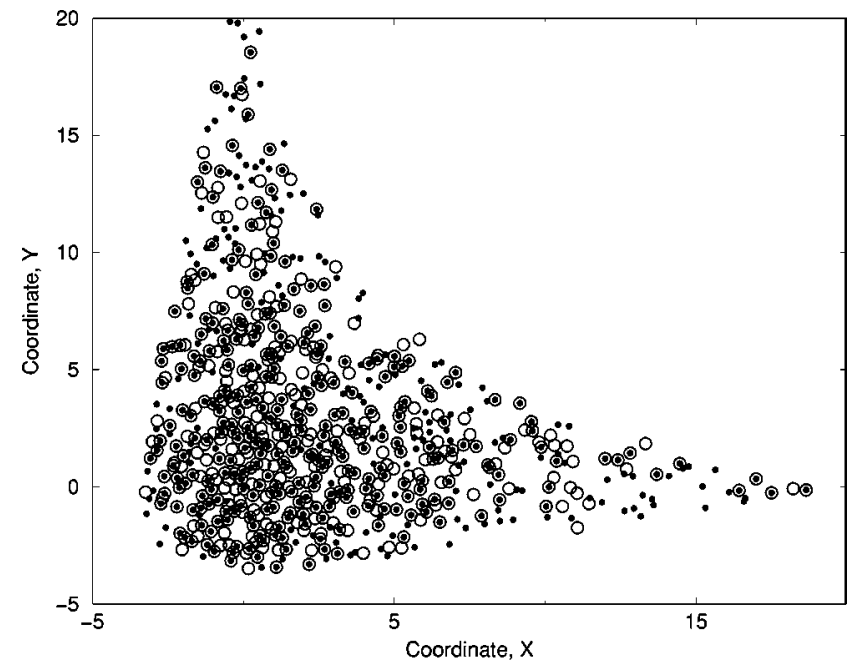

FIG. 8. The positions of 482 basis set functions for nonuniform QDGB with $\gamma=1$ (open circles) and $\gamma=1 / 2$ (filled circles) for the two-dimensional Morse potential.

the construction of the basis sets depends on the separability of the potential. The values of all of the numerical parameters of the bases are shown in Table I.

First, consider the two-dimensional Hamiltonian,

$$
\begin{aligned}
H= & -\frac{1}{2} \frac{d^{2}}{d x^{2}}-\frac{1}{2} \frac{d^{2}}{d y^{2}}+D\left(\exp \left(-w_{x} x\right)-1\right)^{2} \\
& +D\left(\exp \left(-w_{y} y\right)-1\right)^{2} .
\end{aligned}
$$

The Morse parameters are given in Table I. The dissociation energy is 12 a.u. We calculate 122 energy levels below $E_{\text {cut }}=11.5$ a.u., using 482 basis functions for all four calculations. The positions of the basis function centers for the QDGB's is shown in Fig. 8 for both $\gamma$ values.

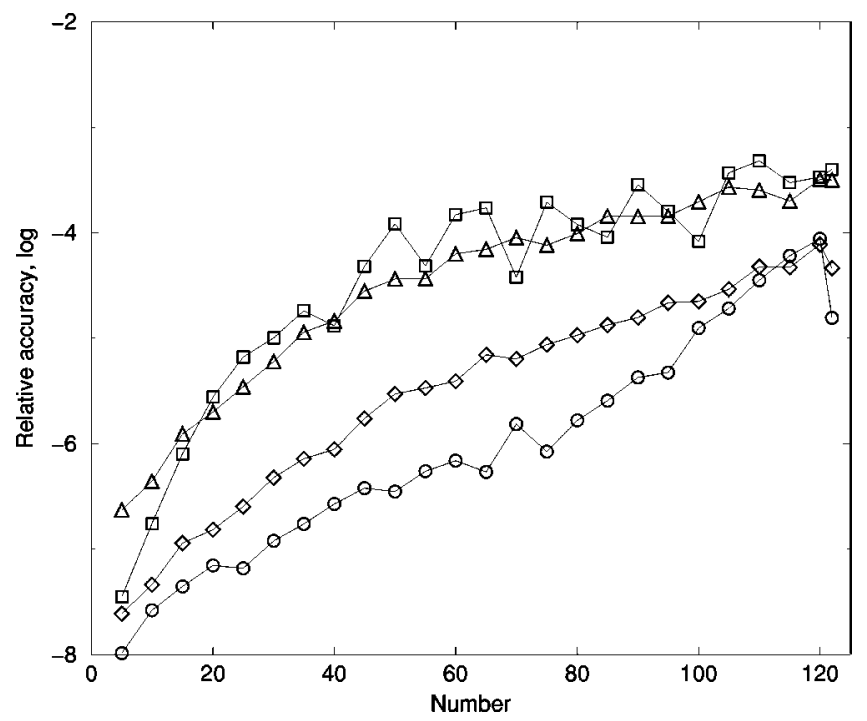

FIG. 9. Logarithm of the relative accuracy of the lowest 122 energy levels, averaged over five levels, for the two-dimensional Morse potential, calculated with 482 Gaussian basis functions distributed on the grid (squares), uniform width QDGB (triangles), variable width QDGB with $\gamma=1$ (circles) and with $\gamma=1 / 2$ as a function of the level number.

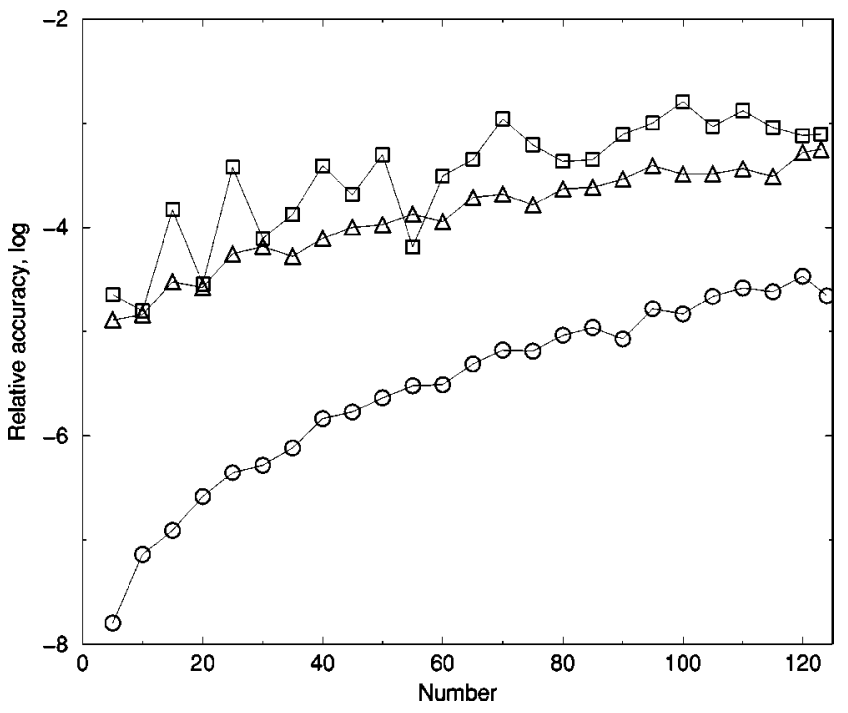

FIG. 10. Logarithm of the relative accuracy of the lowest 124 energy levels, averaged over 5 levels, for the three-dimensional Morse potential, calculated with 939 Gaussian basis functions distributed on the grid (squares), uniformly (triangles), and nonuniformly (circles), on the logarithmic scale as a function of the quantum number.

The logarithm of the relative accuracy is plotted in Fig. 9. The QDGB gives the most accurate results across the whole energy range, with the $\gamma=1$ results being more accurate than the $\gamma=0.5$ results for about the lowest 100 levels.

For the three-dimensional case we consider the following Hamiltonian:

$$
\begin{aligned}
H= & -\frac{1}{2} \frac{d^{2}}{d x^{2}}-\frac{1}{2} \frac{d^{2}}{d y^{2}}-\frac{1}{2} \frac{d^{2}}{d z^{2}}+D\left(\left(\exp \left(-w_{x} x\right)-1\right)^{2}\right. \\
& \left.+\left(\exp \left(-w_{y} y\right)-1\right)+\left(\exp \left(-w_{z} z\right)-1\right)^{2}\right)
\end{aligned}
$$

with parameters shown in Table I The number of energy eigenvalues below $E_{\text {cut }}=7.5$ is 124 . The size of the basis sets is 939 functions. The logarithm of the relative accuracy, averaged over 5 levels, is shown in Fig. 10. The accuracy of the nonuniform basis set is two orders of magnitude better than that of the grid basis for most energy levels. Interestingly, the accuracy given by the uniform quasirandom DGB is somewhat better and more consistent for these two and three-dimensional systems than the results obtained with Gaussians on a grid. The number of basis functions required per accurate eigenvalue scales exponentially with the dimension but with a very small $n: \mathrm{N}_{\text {basis }} \sim \mathrm{N}_{\text {soln }} 2^{d}$.

The 2D Henon-Heiles problem was also solved, with all 99 bound states given by QDGB basis sets of 300 and 462 functions. Accuracy was comparable to that reported by Hamilton ${ }^{8}$ for the same basis size.

\section{CALCULATION OF THE ENERGY LEVELS FOR TRIATOMIC MOLECULES}

\section{A. Water molecule}

To go beyond simple test problems we calculate the vibrational energy levels of water for $J=0$ total angular momentum for an accurate potential energy surface. ${ }^{19}$ Conver- 
gence of the 252 levels below $25118 \mathrm{~cm}^{-1}$ to $0.1 \mathrm{~cm}^{-1}$ was desired and achieved. The triatomic vibrational Hamiltonian in Radau coordinates is

$$
\hat{H}=K^{2 \mathrm{D}}+K^{\theta}+V\left(R_{1}, R_{2}, \theta\right),
$$

where the two-dimensional Hamiltonian is

$$
K^{2 \mathrm{D}}=-\frac{\hbar^{2}}{2 m_{1} R_{1}^{2}} \frac{\partial}{\partial R_{1}}\left(R_{1}^{2} \frac{\partial}{\partial R}\right)-\frac{\hbar^{2}}{2 m_{2} R_{2}^{2}} \frac{\partial}{\partial R_{2}}\left(R_{2}^{2} \frac{\partial}{\partial R_{2}}\right)
$$

and the angular part is

$$
K^{\theta}=-\frac{\hbar^{2}}{2}\left(\frac{1}{m_{1} R_{1}^{2}}+\frac{1}{m_{2} R_{2}^{2}}\right) \mathbf{j}^{2}
$$

with

$$
\mathbf{j}^{2}=\frac{1}{\sin \theta} \frac{\partial}{\partial \theta}\left(\sin \theta \frac{\partial}{\partial \theta}\right) .
$$

The volume element is $R_{1}^{2} R_{2}^{2} \sin \theta d R_{1} d R_{2} d \theta .^{11,20}$

We use the QDGB for the distances $R_{1}$ and $R_{2}$ and the Legendre DVR for the angle $\theta$. This particular form of the Hamiltonian is used in order to calculate all of the kinetic energy operators analytically. Since the potential matrix is diagonal in the DVR with respect to the discretized angle variable, we can construct individual two-dimensional Gaussian bases $\left\{g^{\alpha}\right\}$ for the two-dimensional Hamiltonians for each DVR angle ${ }^{9,20} \theta_{\alpha}$,

$$
H_{j}^{2 \mathrm{D}}=K^{2 \mathrm{D}}+V\left(R_{1}, R_{2}, \theta_{\alpha}\right) .
$$

Two-dimensional Hamiltonians for different $\theta_{\alpha}$ are coupled via $K^{\theta}$ terms with the DVR expression for the $\mathbf{j}^{2}$ operator. $^{21,22}$ The distances $R_{1}$ and $R_{2}$ vary from zero to infinity, and the kinetic energy matrix elements are actually integrated for $R_{1(2)}=[0, \infty]$. A low order Gauss-Hermite quadrature is used to evaluate the potential matrix elements. The 2D basis consists of Gaussians of variable width distributed quasirandomly within the energy contour $E_{\text {cut }}$ as described in Sec. III with the rejection method [Eq. (14)]. The width parameter of $i$ th Gaussian centered at $\left(R_{1 i}, R_{2 i}\right)$ is scaled by masses $m_{1}$ and $m_{2}$,

$$
\alpha_{1(2) i}=c m_{1(2)}\left(E_{\text {cut }}-V\left(R_{1 i}, R_{2 i}\right)+\Delta\right) \text {. }
$$

The "optimal" value of $c$ is found from a one-dimensional minimization (with respect to $c$ ) of the trace of $H^{2 \mathrm{D}}$. In general, one may try a few values of $c$ and choose the value that gives the lowest eigenvalues, instead of this optimization, as was done in Sec. III. The convergence of the energy levels below $E_{\text {cut }}$ is monitored as the basis set is being constructed. The number of Gaussians varies with $\theta_{j}$. The final size of the 2D Hamiltonian matrix is defined by the number of its eigenvalues below the truncation energy $E_{\text {tr }}$. The diagonalized $H_{j}^{2 \mathrm{D}}$ make the diagonal blocks in angle of the full Hamiltonian matrix. The angular part of the Hamiltonian, $K^{\theta}$, is transformed into the bases diagonalizing the $2 \mathrm{D}$ Hamiltonians, is truncated and then is added to the full Hamiltonian matrix. We do not take advantage of the symmetry of the molecule. We calculate energy levels for the $\mathrm{H}_{2} \mathrm{O}$ molecule using the PJT2 potential surface of Polyansky,
TABLE III. Maximal deviation for energy levels of water in $\mathrm{cm}^{-1} . N_{\theta}$ is

\begin{tabular}{|c|c|c|c|c|c|c|c|c|c|c|c|}
\hline \multirow[b]{2}{*}{ Basis } & \multirow[b]{2}{*}{$N_{\theta}$} & \multirow[b]{2}{*}{$N_{q}$} & \multirow[b]{2}{*}{$E_{\mathrm{tr}}$} & \multirow[b]{2}{*}{$E_{\text {cut }}$} & \multirow[b]{2}{*}{$\epsilon^{2 \mathrm{D}}$} & \multirow[b]{2}{*}{$N_{\max }$} & \multicolumn{5}{|c|}{ Number of energy levels } \\
\hline & & & & & & & 400 & 350 & 300 & 250 & 200 \\
\hline I & 43 & 5 & 0.23 & 0.165 & 1.0 & 3551 & & $\cdots$ & & $\cdots$ & \\
\hline II & 39 & 5 & & 165 & 1.0 & & 0.43 & 0.35 & 0.17 & 0.08 & 0.03 \\
\hline III & 43 & 5 & 0.2 & 0.16 & 1.0 & & 1.02 & 0.33 & 0.18 & 0.07 & 0.02 \\
\hline IV & 39 & 5 & 0.2 & 0.16 & 1.0 & & 0.62 & 0.42 & 0.09 & 0.05 & 0.03 \\
\hline $\mathrm{V}$ & 39 & 5 & 0.23 & & 1.0 & 3198 & 0.60 & 0.41 & 0.09 & 0.05 & 0.03 \\
\hline VI & 39 & 7 & 0.22 & & 1.0 & 2838 & 0.64 & 0.43 & 0.11 & 0.07 & 0.03 \\
\hline $\mathrm{VII}^{\mathrm{a}}$ & 39 & 5 & 0.22 & 0.16 & 1.0 & 2777 & 8.38 & 3.76 & 1.90 & 0.50 & 0.09 \\
\hline VIII $^{b}$ & 39 & 5 & 0.22 & 0.16 & 1.0 & 2838 & 0.73 & 0.34 & 0.10 & 0.06 & 0.03 \\
\hline IX & 39 & 5 & & & 2.0 & & & 3.80 & 1.2 & 0.31 & 0.12 \\
\hline$X$ & 39 & 4 & 0.18 & 0.145 & 2.0 & 1574 & 12.29 & 4.70 & 2.50 & 0.87 & 0.24 \\
\hline
\end{tabular}
the number of angular DVR points. $N_{q}$ is the number of quadrature points per dimension for radial integrals. The cutoff energy for the placement of Gaussians, $E_{\text {cut }}$, and the truncation energy for the 2D eigenvalues, $E_{\mathrm{tr}}$ are in hartree. The convergence criterion for energy levels in $2 \mathrm{D}, \epsilon$, is in $\mathrm{cm}^{-1}$. $N_{\max }$ is the total size of the truncated matrix.

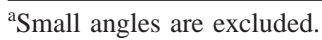

${ }^{\mathrm{b}}$ Different seed for the random sequence in Eq. (15) is used.

Jensen, and Tennyson and compare our results with the band origins published in Ref. 19. The highest published energy level is $25118.31 \mathrm{~cm}^{-1}$. The numerical parameters for 10 different calculations, performed to test the convergence of the results and relative importance of several aspects of the calculation, are listed in Table III. The parameter $\Delta$ of Eq. (23) is 0.01 hartree $\left(\approx 1800 \mathrm{~cm}^{-1}\right)$ for all calculations. The physical constants and conversion factors are given in Table VI.

The typical potential energy cutoff for Gaussian centers is $E_{\text {cut }}=0.16$ hartree $\left(\approx 35000 \mathrm{~cm}^{-1}\right)$ and the truncation energy for 2D Hamiltonian eigenvalues is $E_{\mathrm{tr}}=0.22$ hartree $\left(\approx 48000 \mathrm{~cm}^{-1}\right)$. The number of angular DVR points is 39 .

The 2D QDGB basis is increased until the accuracy of the $2 \mathrm{D}$ eigenvalues below $E_{\text {cut }}$ is $\epsilon=1.0 \mathrm{~cm}^{-1}$ and the minimum RCN for the $2 \mathrm{D}$ overlap matrix is $\mu=10^{-13}$. The $2 \mathrm{D}$ Gaussians are added in increments of 20 until the convergence criterion is met or until their number exceeds 300 , or

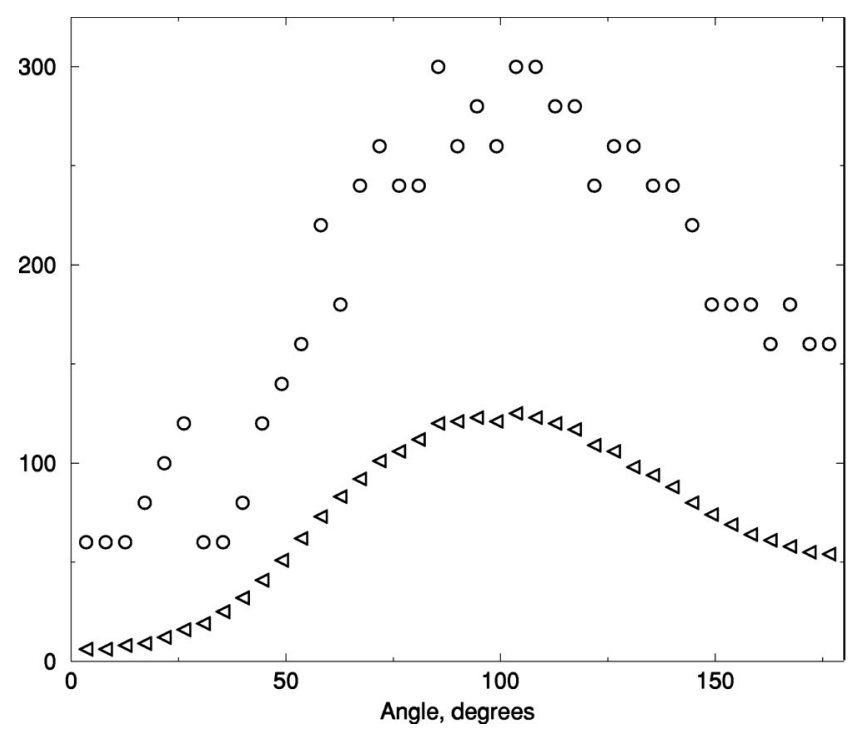

FIG. 11. The size of the 2D quasirandom DGB before and after truncation procedure as a function of angle. 
TABLE IV. The number of levels obtained with the bases II-IV, that are converged to a given accuracy (for all three bases) when compared to the levels of basis I.

\begin{tabular}{lccccc}
\hline \hline $\begin{array}{l}\text { Accuracy, } \mathrm{cm}^{-1} \\
\begin{array}{l}\text { Number } \\
\text { of levels }\end{array}\end{array}$ & 1.02 & 0.50 & 0.20 & 0.10 & 0.05 \\
$\begin{array}{l}\text { Maximal energy, } \\
\mathrm{cm}^{-1}\end{array}$ & 29785 & 28908 & 27357 & 26489 & 23552 \\
\hline \hline
\end{tabular}

until the RCN criterion is not satisfied. In Fig. 11 we show at each angle the number of Gaussians and the number of basis functions kept after truncation of the 2D calculations with basis IV. About $40 \%$ of the energy levels were retained after the truncation procedure. For several angles the convergence criterion for the $2 \mathrm{D}$ eigenvalues of $1 \mathrm{~cm}^{-1}$ is not met, but the convergence of all retained levels below $32000 \mathrm{~cm}^{-1}$ is better than $1.3 \mathrm{~cm}^{-1}$ and convergence of all the levels below $30000 \mathrm{~cm}^{-1}$ is better than $0.55 \mathrm{~cm}^{-1}$.

We have found that keeping Gaussians for all angles, even though $V\left(R_{1}, R_{2}, \theta\right)>E_{\text {cut }}$ for $\theta<39^{\circ}$, has improved the convergence, since these 2D Hamiltonians have a few eigenvalues below $E_{\mathrm{tr}}$. In practice, we raised $E_{\text {cut }}$ for small angles until at least 40 Gaussians were generated.

We also have checked the dependence of the results on the initial seed of the random sequence of the rejection method, the number of quadrature points for $R_{1}$ and $R_{2}$ and on the eigenvalue truncation energy parameter. Parameters and results for two smaller calculations, yielding convergence of band origins under $0.5 \mathrm{~cm}^{-1}$ and $1.0 \mathrm{~cm}^{-1}$ are also included in Table III. Table III shows the maximal difference of the energy levels in comparison with the largest (basis I) calculation for $M$ levels. $M=400,350,300,250$, and 200 correspond to energies of $29520,28183,26714,25069$, and $23153 \mathrm{~cm}^{-1}$, respectively. The number of levels ob- tained with the bases II, III, and IV that differ from the levels of the largest calculation by less than 1.0, 0.5, 0.2, 0.1, and $0.05 \mathrm{~cm}^{-1}$ is shown in Table IV. The total matrix size varies from 1574 to 3551 without taking symmetry into account. This is approximately the same as in Ref. 19 (1000-2000 functions for each of the two symmetries).

Comparison of our results to the energy levels of Ref. 19 is shown in Table V. There is a systematic discrepancy with the results of Polyansky, Jensen, and Tennyson that varies linearly with energy reaching $-0.3 \mathrm{~cm}^{-1}$ for the highest levels. This may be explained by a difference in the energy conversion factor. Levels $n=6$ and $n=39$ differ by more than $1 \mathrm{~cm}^{-1}$, which is apparently due to typographical errors in Ref. 19. Their highest energy level, $n=252$ is about 0.6 $\mathrm{cm}^{-1}$ higher than the present calculation with the systematic discrepancy taken into account. This appears to be a variational error.

We also can compare the performance of the QDGB with the earlier work of Bacic, Watt, and Light ${ }^{11}$ for the $\mathrm{H}_{2} \mathrm{O}$ system, in which a distributed Gaussian basis was used. The location of the basis functions was chosen non-uniformly according to semiclassical considerations, but their width was potential-independent. The full size of the basis in Ref. 11 , if adjusted for the difference in the number of angular points, was approximately the same as in our basis $\mathrm{X}$, and the energy levels under $27000 \mathrm{~cm}^{-1}$ were calculated with the reported convergence of about $5-8 \mathrm{~cm}^{-1}$. Our calculation with the basis $\mathrm{X}$ gave the energy levels to $1 \mathrm{~cm}^{-1}$ accuracy. Although our calculation is performed with a different potential surface and larger number of angles, we believe that the QDGB with its potential-dependent width and density is responsible for superior accuracy of the present calculation.

TABLE V. Energy levels for $\mathrm{H}_{2} \mathrm{O} .\left(d E^{1}=E^{\text {present }}-E^{P J T 2}, d E^{2}=E^{\text {present }}-E^{\text {experimental }}, E^{P J T 2}\right.$-energies from Ref. 19.)

\begin{tabular}{rrrrrrrrrrrr}
\hline \hline $\mathrm{N}$ & $E^{\exp }$ & $d E^{1}$ & \multicolumn{1}{c}{$d E^{2}$} & $\mathrm{~N}$ & $E^{\exp }$ & $d E^{1}$ & $d E^{2}$ & $\mathrm{~N}$ & $E^{\exp }$ & $d E^{1}$ & $d E^{2}$ \\
\hline 2 & 1594.6 & -0.04 & -0.06 & 28 & 10600.7 & -0.17 & 1.00 & 69 & 15348.0 & -0.26 & 0.06 \\
3 & 3151.5 & -0.07 & -0.14 & 29 & 10613.7 & -0.17 & 0.27 & 72 & 15742.6 & -0.26 & -0.21 \\
4 & 3657.1 & -0.06 & -0.01 & 30 & 10868.4 & -0.19 & -0.46 & 74 & 15833.0 & -0.29 & 0.24 \\
5 & 3755.8 & -0.03 & -0.13 & 31 & 11032.5 & -0.22 & -0.25 & 84 & 16822.6 & -0.29 & 0.02 \\
6 & 4666.8 & -1.10 & -0.01 & 36 & 11812.4 & -0.23 & -0.76 & 85 & 16823.4 & -0.27 & -1.84 \\
7 & 5234.9 & -0.10 & -0.11 & 38 & 12139.5 & -0.21 & 0.62 & 86 & 16897.9 & -0.26 & -0.53 \\
8 & 5331.3 & -0.11 & 0.04 & 39 & 12151.1 & -1.22 & -0.22 & 87 & 16898.0 & -0.26 & -0.76 \\
9 & 6133.0 & -0.13 & -0.97 & 41 & 12406.8 & -0.23 & -0.85 & 91 & 17227.2 & -0.29 & -0.50 \\
10 & 6775.1 & -0.12 & 0.03 & 43 & 12565.2 & -0.24 & 0.24 & 92 & 17313.5 & -0.31 & 0.97 \\
11 & 6871.5 & -0.14 & -0.03 & 49 & 13642.6 & -0.24 & 0.38 & 96 & 17460.4 & -0.27 & 2.08 \\
12 & 7202.1 & -0.12 & 0.61 & 50 & 13652.6 & -0.24 & 0.40 & 97 & 17496.6 & -0.29 & 1.10 \\
13 & 7249.8 & -0.13 & 0.00 & 53 & 13829.1 & -0.22 & 0.77 & 99 & 17750.5 & -0.30 & 2.36 \\
14 & 7444.6 & -0.14 & -0.49 & 54 & 13831.2 & -0.23 & 0.31 & 110 & 18393.6 & -0.30 & 0.62 \\
16 & 8273.8 & -0.16 & -0.24 & 55 & 13910.1 & -0.25 & -0.77 & 111 & 18394.1 & -0.30 & 0.81 \\
17 & 8373.9 & -0.17 & 0.06 & 56 & 14066.7 & -0.27 & 0.49 & 119 & 18991.7 & -0.32 & 1.76 \\
18 & 8761.7 & -0.15 & 0.68 & 57 & 14221.7 & -0.23 & 0.51 & 132 & 19780.4 & -0.27 & -0.65 \\
19 & 8806.7 & -0.16 & -0.28 & 58 & 14319.3 & -0.26 & 0.49 & 146 & 20545.2 & -0.32 & 2.14 \\
21 & 9000.0 & -0.18 & -0.14 & 59 & 14538.6 & -0.27 & 1.66 & 158 & 21222.9 & -0.32 & -0.71 \\
23 & 9832.6 & -0.20 & -1.03 & 65 & 15107.1 & -0.27 & 0.10 & 159 & 21223.4 & -0.32 & -0.32 \\
25 & 10284.5 & -0.18 & 0.06 & 66 & 15118.6 & -0.27 & -0.35 & 187 & 22528.6 & -0.27 & -0.78 \\
26 & 10328.4 & -0.20 & -0.32 & 68 & 15345.3 & -0.26 & 0.81 & 252 & 25117.3 & -0.97 & -1.06 \\
\hline \hline
\end{tabular}


TABLE VI. Values of the physical constants and conversion factors used in the calculation.

\begin{tabular}{lc}
\hline \hline Relative mass of $\mathrm{O}^{16}$ & 15.99491464 \\
Relative mass of $H$ & 1.007825037 \\
Conversion to atomic units of mass & 1822.88853006 \\
Conversion from hartree to $\mathrm{cm}^{-1}$ & 219474.629 \\
Conversion from bohr to $\AA$ & 0.529177249 \\
\hline \hline
\end{tabular}

\section{B. Neon and argon trimers}

To test the effectiveness of QDGB we also calculate several low-lying energy levels of $\mathrm{Ne}_{3}$ and $\mathrm{Ar}_{3}$ following Ref. 15 using a 3D QDGB. The Hamiltonian of a trimer has a simple form in the atom-atom pair coordinates $R_{1}, R_{2}$, and $R_{3}$

$$
\begin{aligned}
H= & \sum_{i=1}^{3}\left\{-\frac{\hbar^{2}}{m}\left[\frac{1}{R_{i}^{2}} \frac{\partial}{\partial R_{i}} R_{i}^{2} \frac{\partial}{\partial R_{i}}+\frac{R_{j}^{2}+R_{k}^{2}-R_{i}^{2}}{2 R_{j} R_{k}} \frac{\partial^{2}}{\partial R_{j} \partial R_{k}}\right]\right. \\
& \left.+V\left(R_{i}\right)\right\}, \quad i \neq j \neq k .
\end{aligned}
$$

The distances $R_{1}, R_{2}$, and $R_{3}$ must satisfy the triangle inequality,

$$
\left|R_{3}-R_{1}\right| \leqslant R_{2} \leqslant R_{1}+R_{3} .
$$

The volume element is

$$
d \Omega=R_{1} R_{2} R_{3} d R_{1} d R_{2} d R_{3} .
$$

The potential is

$$
V\left(R_{i}\right)=D\left(\exp \left(-\alpha\left(R_{i}-R_{e}\right)\right)-1\right)^{2} .
$$

The parameter values are $D=99.0 \mathrm{~cm}^{-1}, \alpha=1.72 \AA^{-1}$, and $R_{e}=3.757 \AA$ for $\mathrm{Ar}$ and $D=29.36 \mathrm{~cm}^{-1}, \alpha$ $=2.088 \AA^{-1}$, and $R_{e}=1.717 \AA$ for Ne.

Unlike the standard Jacobi or hyperspherical coordinates the pair coordinates are nonorthogonal, but they have the advantage that the symmetry of the system can be easily imposed. We construct a symmetrized basis out of quasirandom Gaussians in the following way. The centers of Gaussians with the potential-adapted width and density are chosen as before, except that now $k$ th Gaussian center $\left(R_{1}^{k}, R_{2}^{k}, R_{3}^{k}\right)$ satisfies the condition $R_{1}^{k}<R_{2}^{k}<R_{3}^{k}$ and is omitted from further considerations if it does not satisfy the triangle inequality. Then, five more Gaussians are obtained by permutation: $\left(R_{2}^{k}, R_{3}^{k}, R_{1}^{k}\right),\left(R_{2}^{k}, R_{1}^{k}, R_{3}^{k}\right)$ and so on. The final symmetrized basis function is a normalized sum of the $k$ th Gaussian and its permutations. This generates the basis for $A_{1}$ symmetry appropriate for ${ }^{40} \mathrm{Ar}_{3}$ and ${ }^{20} \mathrm{Ne}_{3}$ (Table VI).

For comparison purposes we computed the energy levels reported in Ref. 15 (six for $\mathrm{Ar}_{3}$ and two for $\mathrm{Ne}_{3}$ ) to accuracies comparable to or better than reported earlier using small QDGB's. For $\mathrm{Ar}_{3}$ the six lowest states were obtained within $0.5 \mathrm{~cm}^{-1}$ accuracy with respect to the converged energy levels (below), using 70 symmetrized basis functions. In Ref. 15 286 symmetrized DGB's were used. The energy levels are listed in Table VII. The QDGB parameters of Eq. (13) were $E_{\text {cut }}=114.0 \mathrm{~cm}^{-1}, \Delta=30.0 \mathrm{~cm}^{-1}, c=0.01$. The range of Gaussian centers was from $3.37 \AA$ to $5.53 \AA$ in all coordi-
TABLE VII. The lowest energy levels for $\mathrm{Ar}_{3}$ obtained using the equidistant DGB (taken from Ref. 15) and using the QDGB in $\mathrm{cm}^{-1}$. The QDGB ${ }^{\dagger}$ is the largest basis that was used to calculate all bound states (see the details in the text).

\begin{tabular}{rccc}
\hline \hline $\begin{array}{c}\text { Basis } \\
\text { Size }\end{array}$ & $\begin{array}{c}\text { DGB } \\
286\end{array}$ & $\begin{array}{c}\text { QDGB } \\
70\end{array}$ & $\begin{array}{c}\text { QDGB }^{\dagger} \\
400\end{array}$ \\
\hline 1 & 44.57 & 44.57 & 44.57 \\
2 & 76.09 & 76.10 & 76.08 \\
3 & 88.83 & 88.82 & 88.78 \\
4 & 103.55 & 103.40 & 103.28 \\
5 & 108.20 & 108.07 & 107.96 \\
6 & 116.88 & 116.07 & 115.62 \\
7 & $\ldots$ & $\ldots$ & 119.76 \\
8 & $\ldots$ & $\ldots$ & 125.33 \\
9 & $\ldots$ & $\ldots$ & 128.37 \\
10 & $\ldots$ & $\cdots$ & 129.28 \\
\hline \hline
\end{tabular}

nates. The matrix elements were calculated using the 5-point Gauss-Hermite quadrature with the infinite limits of integration. The size of the QDGB in our calculation was four times smaller than that in Ref. 15, where Gaussian centers were equidistant. For $\mathrm{Ne}_{3}$, following Ref. 15, we computed two lowest energy levels using 150 Gaussians, whose centers were distributed between $2.63 \AA$ and $6.64 \AA$. The rest of the QDGB parameters were $E_{\text {cut }}=75.0 \mathrm{~cm}^{-1}, \Delta=10.0$ $\mathrm{cm}^{-1}, c=0.007$, and the number of quadrature points per dimension was 7. The values of the energy levels are $E^{0}$ $=-50.54 \mathrm{~cm}^{-1}$ and $E^{1}=-34.67 \mathrm{~cm}^{-1}$, which are lower than those reported in Ref. 15 by 0.3 and $0.8 \mathrm{~cm}^{-1}$, respectively. The basis size in this calculation is also more than 4 times smaller than that of Ref. 15.

We also tried to compute the energy levels up to the dissociation limit $\left(208 \mathrm{~cm}^{-1}\right)$ for $\mathrm{Ar}_{3}$ for this potential and found that the convergence above the isomerization limit [130 $\mathrm{cm}^{-1}$ (Ref. 23)] was poor. The converged ten lower states are presented in the last column of Table VII, labeled QDGB $^{\dagger}$. We used 400 symmetrized Gaussians, distributed between $3.36 \AA$ and $8.17 \AA$. The parameters of Eq. (13) were $E_{\mathrm{cut}}=135 \mathrm{~cm}^{-1}, \Delta=10.0 \mathrm{~cm}^{-1}$, and $c=0.012$. We used seven quadrature points per dimension to compute the matrix elements. The lower eight states are converged within $0.02 \mathrm{~cm}^{-1}$ and the two remaining states are converged to about $0.1 \mathrm{~cm}^{-1}$. We believe that at the energies above the isomerization barrier the collinear configuration of $\mathrm{Ar}_{3}$ becomes important and the eigenfunction amplitudes are nonzero at the triangle inequality boundaries. Thus, proper (zero derivative) boundary conditions on the basis functions at the triangle inequality and finite integration limits have to be imposed in order to obtain the higher energy states accurately.

\section{SUMMARY}

The size of direct product basis sets scales exponentially with the number of degrees of freedom. Thus, the use of these basis sets becomes less efficient with the increase of the dimensionality of systems. For such problems a multicenter correlated basis is desirable. This is confirmed by the results of multidimensional localized functions distributed within an energy contour on the potential surface. In this 
paper we have proposed a quasirandom distributed Gaussian basis (QDGB) that is well suited for multidimensional vibrational problems. The advantage of this basis is that it is adapted to a given potential and is correlated by construction, which makes it efficient in many dimensions. The localized character of the Gaussians requires low order quadratures for the potential matrix evaluations. The simplicity of generating this basis and the ability to check the convergence of eigenvalues during its construction are also advantages for multidimensional problems when the feasibility and the numerical effort is ultimately defined by the size of the basis set. A QDGB can be readily combined with a DVR in angle (the DGB-DVR approach), ${ }^{9-12}$ yielding a "customized"' basis for each DVR angle. Although a DVR-DGB approach was used before, the efficiency and accuracy is substantially improved using QDGB's as demonstrated in our calculation of the $J=0$ levels of $\mathrm{H}_{2} \mathrm{O}$.

We also believe that QDGB can be useful for systems, that are most conveniently represented in nonorthogonal pair coordinates. As we have shown for $\mathrm{Ne}_{3}$ and $\mathrm{Ar}_{3}$, the proper symmetry can be easily imposed on QDGB. The final QDGB size required for the lower levels was four times smaller than in the earlier calculation ${ }^{15}$ of comparable accuracy.

\section{ACKNOWLEDGMENTS}

This research was supported in part by a grant from the Department of Energy, DE-FG02-87ER13679.

${ }^{1}$ J. V. Lill, G. A. Parker, and J. C. Light, Chem. Phys. Lett. 89, 483 (1982).

${ }^{2}$ J. C. Light, I. P. Hamilton, and J. V. Lill, J. Chem. Phys. 82, 1400 (1985).

${ }^{3}$ J. C. Light and T. Carrington, Jr., Adv. Chem. Phys. 114, 263 (2000).

${ }^{4}$ A. Viel and C. Leforestier, J. Chem. Phys. 112, 1212 (2000).

${ }^{5}$ R. A. Friesner, J. Chem. Phys. 99, 324 (1993).

${ }^{6}$ H. Chen, S. Liu, and J. C. Light, J. Chem. Phys. 110, 168 (1999).

${ }^{7}$ M. J. Davis and E. J. Heller, J. Comput. Phys. 71, 3383 (1979).

${ }^{8}$ I. P. Hamilton and J. C. Light, J. Chem. Phys. 84, 306 (1986).

${ }^{9}$ Z. Bacic and J. C. Light, J. Chem. Phys. 85, 4594 (1986).

${ }^{10}$ Z. Bacic, R. M. Whitnell, D. Brown, and J. C. Light, Comput. Phys. Commun. 51, 35 (1988).

${ }^{11}$ Z. Bacic, D. Watt, and J. C. Light, J. Chem. Phys. 89, 947 (1988).

${ }^{12}$ Z. Bacic and J. C. Light, Annu. Rev. Phys. Chem. 40, 469 (1989).

${ }^{13}$ M. Mladenovic and S. Schmatz, J. Chem. Phys. 109, 4456 (1998).

${ }^{14}$ X. G. Hu, T. S. Ho, and H. Rabitz, Phys. Rev. E 61, 2074 (2000).

${ }^{15}$ T. Gonzalez-Lezana, J. Rubayo-Soneira, S. Miret-Artes, F. A. Gianturco, G. Delgado-Barrio, and P. Villarreal, J. Chem. Phys. 110, 9000 (1999).

${ }^{16}$ A. C. Peet, J. Chem. Phys. 90, 4363 (1989).

${ }^{17}$ B. Poirier and J. C. Light, J. Chem. Phys. 113, 211 (2000).

${ }^{18}$ W. Press, B. Flannery, S. Teukolsky, and W. Vetterling, Numerical Recipes: The Art of Scientific Computing, 2nd ed. (Cambridge University Press, Cambridge, 1992).

${ }^{19}$ O. L. Polansky, P. J. Jensen, and J. Tennyson, J. Chem. Phys. 105, 6490 (1996).

${ }^{20}$ S. E. Choi and J. C. Light, J. Chem. Phys. 97, 7031 (1992).

${ }^{21}$ Z. Bacic and J. C. Light, J. Chem. Phys. 86, 3065 (1987).

${ }^{22}$ J. C. Light and Z. Bacic, J. Chem. Phys. 87, 4008 (1987).

${ }^{23}$ N. J. Wright and J. M. Hutson, J. Chem. Phys. 110, 902 (1999). 